\title{
LA ATENCIÓN PRENATAL EN TIEMPOS DE COVID-19
}

\section{PRENATAL CARE IN TIMES OF COVID-19}

\author{
Enrique Guevara Ríos $1,2, a$
}

EI SARS-COV-2 es una nueva cepa de coronavirus, identificada por primera vez en Wuhan, provincia de Hubei, en China a finales del 2019, donde se detectó el primer caso de neumonía.

El 6 de Marzo de 2020 se anunció el paciente cero en nuestro país y el 11 de marzo de 2020 el Director General de la Organización Mundial de la Salud OMS, el doctor Tedros Adhanom Ghebreyesus, anunció que la nueva enfermedad por el coronavirus 2019 (COVID-19) puede caracterizarse como una pandemia.

La epidemia de COVID-19 fue declarada por la OMS una emergencia de salud pública de preocupación internacional el 30 de enero de 2020. La caracterización de pandemia significa que la epidemia se ha extendido por varios países, continentes o todo el mundo, y que afecta a un gran número de personas ${ }^{1}$.

El gobierno y el Ministerio de Salud, tomaron una serie de medidas para enfrentar a la pandemia, entre ellas estaba el uso obligatorio de mascarillas, el aislamiento social, el lavado de manos y un estado de cuarentena en todo el país. Esto trajo como consecuencia el cierre de los consultorios externos en todos los establecimientos de salud públicos y privados, lo que incluía la atención prenatal.

La atención prenatal es una oportunidad decisiva para que los profesionales sanitarios brinden atención, apoyo e información a las embarazadas. Ello incluye la promoción de un modo de vida sano, incluida una buena nutrición, la detección y la prevención de enfermedades, la prestación de asesoramiento para la planificación familiar y el apoyo a las mujeres que puedan estar sufriendo violencia de pareja².

Las gestantes desde marzo del 2020, no han tenido la posibilidad de tener acceso a la atención prenatal. Sin embargo, en el Instituto Nacional Materno Perinatal, se estableció mecanismos para brindar orientación clara y sencilla a toda gestante, familia y comunidad, reforzando la identificación de signos de alarma (fiebre / tos I dificultad para respirar); las comunicaciones sobre la condición médica y las posibles complicaciones se realizaron mediante el WhatsAPP, entre las gestantes y los médicos gineco-obstetras del instituto. Para ello se brindó a la familia un número telefónico de emergencia del equipo de salud para consultas ${ }^{3,4}$.

Se tomó en cuenta las experiencias del seguimiento remoto o presencial dependiendo del riesgo obstétrico en Francia ${ }^{5}$, y del Columbia University Irving Medical Center de Nueva York que reportó la individualización de la atención según los factores de riesgo de la paciente incorporando a la telesalud las recomendaciones de la Academia Americana de Gíneco Obstetricia ${ }^{6}$.

Se construyó un modelo mixto de atención con visitas virtuales y presenciales. Se usó un aplicativo de teléfono móvil para las citas virtuales y se entrenó previamente a los proveedores. La Resolución Directoral 099-2020-DG-INMP-MINSA del 30 de abril, aprobó el Proyecto de TELEORIENTACIÓN para pacientes de bajo riesgo y atención presencial restringida para pacientes con riesgo de complicación. Entre mayo y setiembre del 2020 se habían atendido 5596 teleconsultas y 638 consultas presenciales. Este proyecto toma como base la inversión de la pirámide del control prenatal propuesta por el Servicio de Medicina Fetal del INMP y que permiten la detección de las gestantes de alto riesgo para preeclampsia, parto pretérmino, restricción de crecimiento intrauterino, placenta anormal, detección de malformaciones congénitas, entre otros.

\footnotetext{
Instituto Nacional Materno Perinatal. Lima, Perú.

2 Departamento de Ginecología y Obstetricia. Universidad Nacional Mayor de San Marcos. Lima,Perú.

a Médico Ginecólogo-Obstetra. Director del Instituto Nacional Materno Perinatal. Coordinador de Asistencia Técnica del Instituto de Salud Popular. Consultor de Pathfinder International. Profesor Ordinario de la Facultad de Medicina, Universidad Nacional Mayor de San Marcos Lima-Perú.

Citar como: Guevara R. La atención prenatal en tiempos de COVID-19. Rev Peru Investig Matern Perinat 2020; 9(3): 6-7 DOI https://doi.org/10.33421/inmp.2020208
} 
De esta manera el INMP viene contribuyendo a mantener la atención prenatal, que durante muchos años ha sido una de las principales estrategias junto con el parto institucional para disminuir la mortalidad materna en el Perú; y que aún en épocas de pandemia se pueda brindar a las gestantes para mejorar la calidad de vida materna y neonatal.

Financiamiento: Autofinanciado.

Conflicto de interés: El autor declara no tener algún conflicto de interés.

\section{REFERENCIAS BIBLIOGRÁFICAS}

1. https://www.paho.org/es/noticias/11-3-2020-oms-caracteriza-covid-19-como-pandemia.

2. https://www.who.int/es/news/item/07-11-2016-pregnant-women-must-be-able-to-access-the-right-care-at-the-right-timesays-who.
3. RM 217-2020 MINSA Directiva sanitaria para garantizar la salud de la gestante y la continuidad de planificación familiar ante la infección de COVID 19.

4. Guía provisional para establecimientos de salud: preparación para la transmisión comunitaria de COVID-19 en los Estados Unidos. Colegio Estadounidense de Obstetras y Ginecólogos y la Sociedad de Medicina Materno-Fetal. ACOG 18 marzo 2020.

5. Vivanti AJ, et al. Follow-up for pregnant women during the COVID-19 pandemic: French national authority for health recommendations. J Gynecol Obstet Hum Reprod. 2020:101804.

6. Aziz A, et al. Telehealth for High-Risk Pregnancies in the Setting of the COVID-19 Pandemic. Am J Perinatoly. 2020. 